\title{
Surgical Management of Compound Odontome Associated with Impacted Canine: A Case Report
}

\author{
Manjakandy Smitha ${ }^{1}$, Santhosh T Paul ${ }^{2}$, Vivek Bagga ${ }^{3}$
}

\begin{abstract}
Odontomas are asymptomatic bony lesions that often lead to delayed eruption/impaction of the associated tooth and are detected as an accidental finding on radiographic examination when there is delayed eruption of the permanent tooth, usually in the second decade of life. Most frequently impacted teeth by odontomas are canine, maxillary central incisors, and wisdom tooth. This is a case report of a female patient aged 12 years, with a chief complaint of painless swelling in the left maxillary anterior region, associated with the retained primary canine. On radiographic examination, multiple denticles were found hindering the path of eruption of the permanent canine. The treatment protocol involved surgical removal of the odontome to facilitate the unhindered eruption of the permanent canine into its normal position in the oral cavity. Early identification of odontomas enables simple management, cost-effective treatment, and better prognosis.

Keywords: Compound odontome, Denticles, Hamartomat, Impacted tooth, Radio-opacitics.

Journal of South Asian Association of Pediatric Dentistry (2019): 10.5005/jp-journals-10077-3026
\end{abstract}

\section{INTRODUCTION}

Odontomas are lesions of the odontogenic origin consisting of enamel, dentin, cementum, and pulpal tissue, which are nonaggressive hamartomatous developmental malformation. ${ }^{1}$ In 1867, Paul Broca ${ }^{2}$ coined and defined the term "odontoma" as tumors formed by the overgrowth of transitory dental tissues. ${ }^{3}$ Odontomas are usually asymptomatic and seen most commonly in association with unerupted teeth during routine radiographic examination. Since odontomas are composed of various tooth-like structures that can undergo cystic changes, they may lead to bone expansion and interference in the eruption of the tooth. If an odontome is undiagnosed and left untreated, it can result in complications like impaction/delayed eruption of succedaneous teeth, retention of deciduous teeth, pain, tooth displacement, paresthesia, and swelling of the affected area. Due to these complications, patient's esthetics and functions can be compromised. , $^{3,4}$

The present case report describes the importance of early identification of odontomas that reduces the possibility of development of any pathology/malocclusion in the affected region, thereby enabling minimally expensive treatment and better prognosis.

\section{Case Description}

A female patient aged 12 years reported to the Department of Paedodontics and Preventive Dentistry, with a chief complaint of mild swelling in relation to the upper left front tooth region since 2 months, which was asymptomatic (Fig. 1). The medical and family history was insignificant. All other vital signs were normal. No abnormalities were observed extraorally. Intraoral examination revealed a mixed dentition period. Labial marginal gingiva was inflamed from the 22 to 25 regions. Bony prominence was observed toward the labial cortical plate, apical and mesial to the 63 region measuring $1 \times 1 \mathrm{~cm}$ (Fig. 2). The panoramic and intraoral periapical radiographs (IOPAR) revealed multiple tooth-like radio-opacities (denticles) contained within a fine radiolucent rim apical and mesial to the 63 region (Figs 3 and 4). Unerupted canine was located
1,2Department of Paedodontics and Preventive Dentistry, Sri Rajiv Gandhi College of Dental Sciences and Hospital, Bengaluru, Karnataka, India

${ }^{3}$ Department of Oral and Maxillofacial Surgery, Dental Clinic, Hosmat Hospital, Bengaluru, Karnataka, India

Corresponding Author: Manjakandy Smitha, Department of Paedodontics and Preventive Dentistry, Sri Rajiv Gandhi College of Dental Sciences and Hospital, Bengaluru, Karnataka, India, Phone: +91 9845193394, e-mail: drsmithjuno@gmail.com

How to cite this article:Smitha Manjakandy, Paul ST, BaggaV. Surgical ManagementofCompoundOdontomeAssociated withlmpactedCanine:ACase Report. J South Asian Assoc Pediatr Dent 2019;2(2):64-68.

Source of support: Nil

Conflict of interest: None

apically to the tooth-like radio-opacities. Since the removal of the odontome in a pediatric patient having mixed dentition under

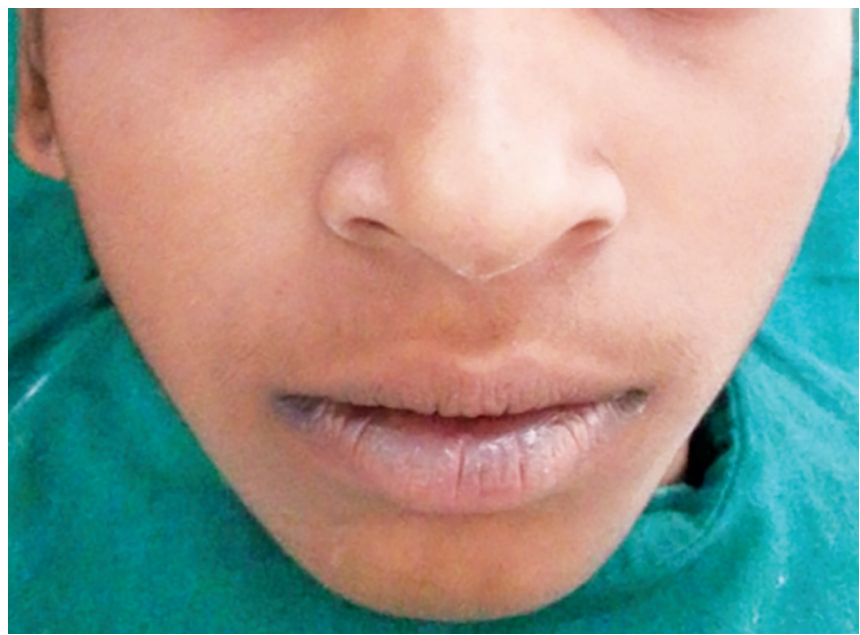

Fig. 1: Mild swelling in relation to the upper left front tooth region

() The Author(s). 2019 Open Access This article is distributed under the terms of the Creative Commons Attribution 4.0 International License (https://creativecommons. org/licenses/by-nc/4.0/), which permits unrestricted use, distribution, and non-commercial reproduction in any medium, provided you give appropriate credit to the original author(s) and the source, provide a link to the Creative Commons license, and indicate if changes were made. The Creative Commons Public Domain Dedication waiver (http://creativecommons.org/publicdomain/zero/1.0/) applies to the data made available in this article, unless otherwise stated. 


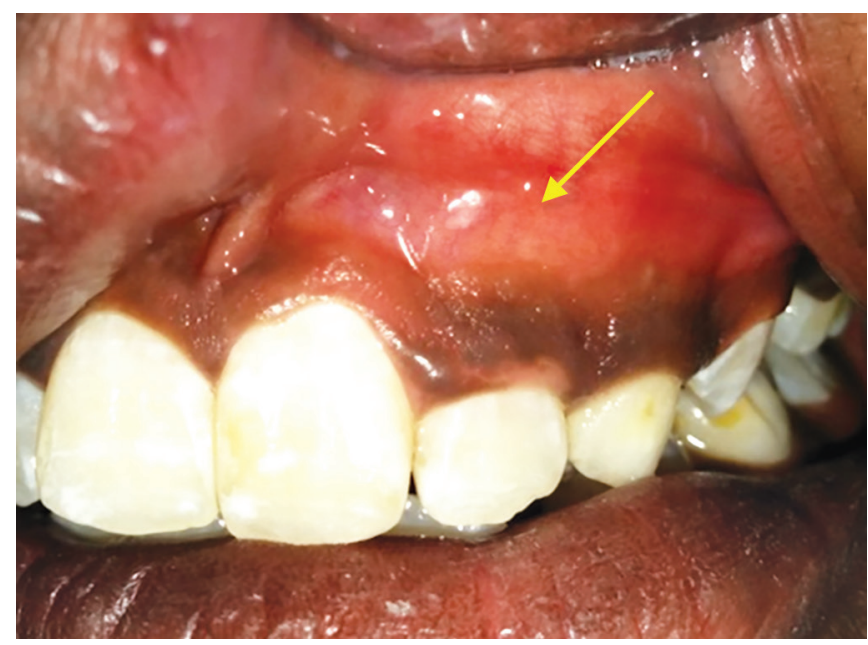

Fig. 2: Bony prominence of the labial cortical plate in the apical and mesial regions of 63 measuring $1 \times 1 \mathrm{~cm}$, the color of the overlying mucosa seen normal

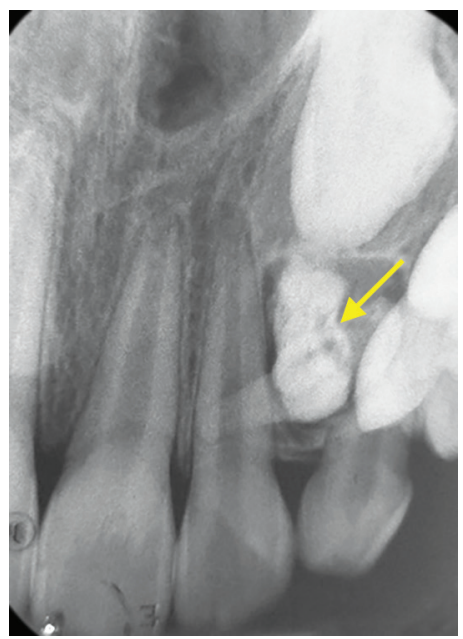

Fig. 4: Intraoral periapical radiograph of the maxillary left region showing an odontome with the apically located impacted canine

local anesthesia (LA) was a challenge for the clinician, advanced 3D cone-beam computed tomography (CBCT), the gold standard for imaging the oral and maxillofacial area, was obtained for accurate localization of the odontome and its relationship with the impacted canine and other neighboring structures (Fig. 5). A provisional diagnosis of the compound odontome was made based on the clinical and radiographic findings. A written informed consent was obtained for the surgical enucleation of the lesion under LA. A window was prepared over the labial cortical bone in relation to the swelling to reveal the multiple denticles (Fig. 6). Tooth-like structures (around eight) were removed with a curette along with the fibrous capsule and both were sent for histopathological examination. Evidence of concrescence, fusion, and dilacerations was noticed and size of denticles varied from 1 to $2 \mathrm{~cm}$ (Fig. 7). Care was taken not to disturb the permanent canine lying apical to it. The debridement of the remnants in the area was done by curettage. After hemostasis, normal saline was used to irrigate the area, repositioning of the mucoperiosteal flap was done by suturing, and the permanent canine was left to erupt naturally into the oral cavity. Histopathological examination of the excised mass confirmed the

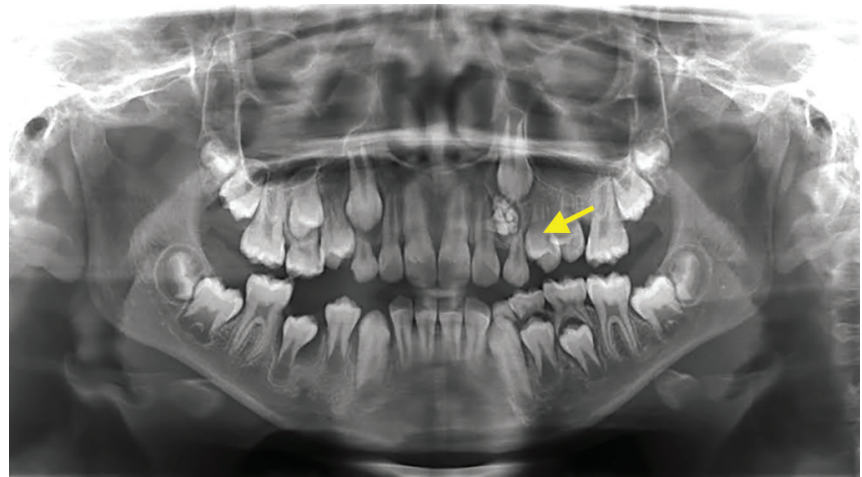

Fig. 3: Panoramic radiograph revealing multiple tiny tooth-like radioopacities (denticles) structures contained within a fine radiolucent rim

diagnosis of the compound odontome (Fig. 8). Orthopantomogram (OPG) was taken after 7 days to evaluate the postoperative healing (Fig. 9). OPG was repeated after 45 days to evaluate the position of 23 and the root formation. There were no significant changes in 23 with respect to its position and root formation was not complete (Fig. 10). So, the patient was put to follow-up for clinical and radiographic examination once in 3 months to evaluate the eruption of 23 and to examine for the recurrence. If the root formation of unerupted 23 completes before eruption, an interdisciplinary approach may be necessary, as the eruptive power is greatly diminished once root formation is completed. Eighteen months of follow-up showed that all the primary teeth had exfoliated and the permanent successors had erupted into their corresponding position in the arch. Also the odontome-associated permanent canine showed significant changes in its position and had erupted in normal alignment in the arch. Radiographic examination revealed a normal trabecular pattern and healing at the surgical site (Fig. 11).

\section{Discussion}

The most common type of odontogenic tumor is odontoma, referred commonly as hamartoma, not a true tumor. Most of the case studies on odontomas recorded worldwide showed odontomas being the most prevalent odontogenic tumor in an interval of $35-76 \% .{ }^{5}$ Mosqueda-Taylor et al. conducted a study on 349 odontogenic tumors in Mexican population and concluded that $35 \%$ comprised odontomas. ${ }^{6}$

Complex odontomas have site predilection to the posterior region of both maxillary and mandibular jaws, whereas in anterior maxilla compound odontomas are more common. ${ }^{7}$ In the literature, about $33 \%$ of odontomas were observed in the mandible and $67 \%$ in the maxilla. It is also reported that complex odontomas had $59 \%$ predilection in posterior jaws and $34 \%$ in anterior maxilla, whereas $61 \%$ of compound odontomas were detected in anterior maxilla. ${ }^{3}$ The present case was in correlation with the above study.

The etiology of the odontoma is not clear, although various factors like local trauma, infection, hereditary anomalies, odontoblastic hyperactivity, or alterations of the genetic components responsible for controlling tooth development has been suggested. ${ }^{8}$ According to WHO classification of odontogenic tumors, compound odontomas are malformations in which the lesion consists of many tooth-like structures with more orderly arrangement of dental tissues. Complex odontomas are malformations with well-formed and less orderly arranged dental tissues. The lesion does not resemble tooth-like structures. It has more growth potential than the compound odontome. ${ }^{7,9}$ 

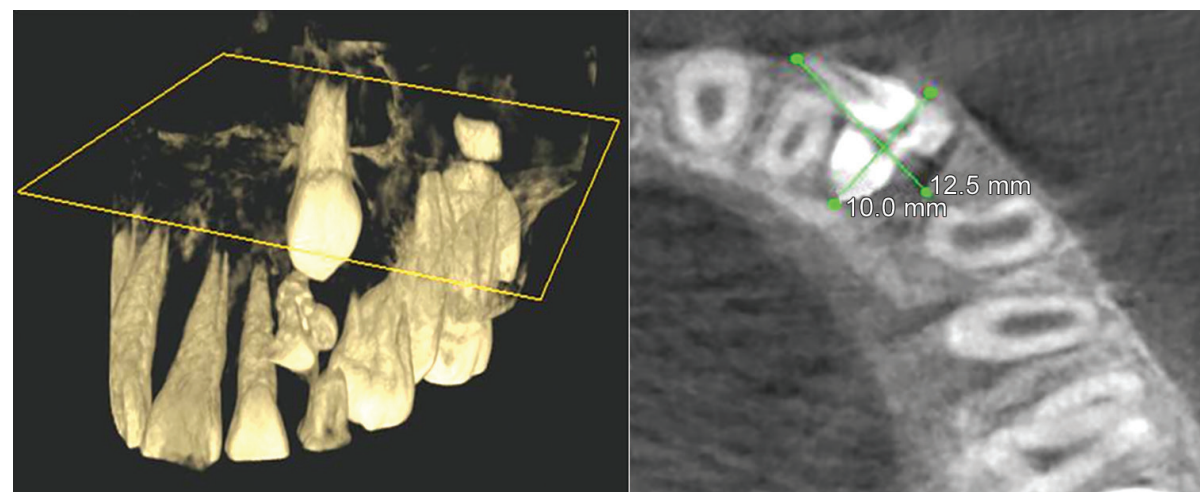

Fig. 5: Cone-beam computed tomography showing the accurate location of the odontome and its relationship with the impacted canine and neighboring structures

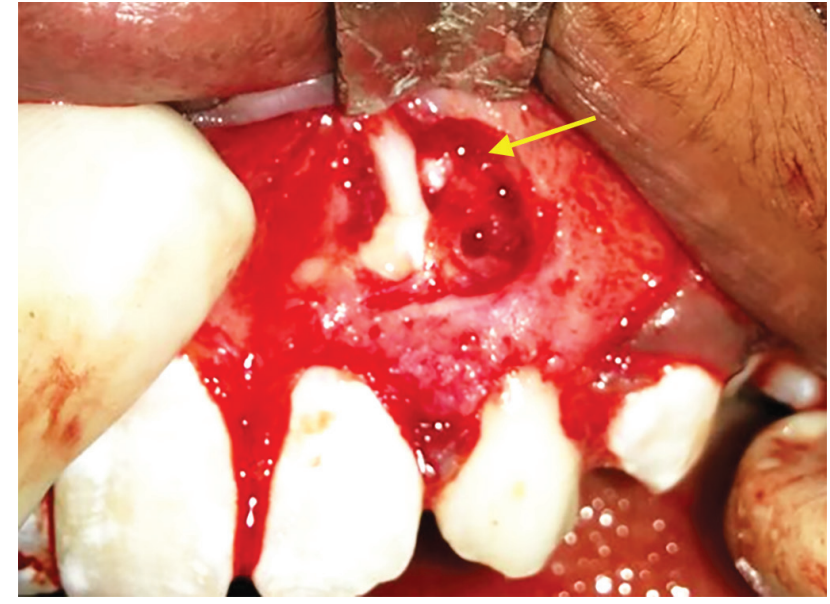

Fig. 6: After reflection of the full-thickness mucoperiosteal flap under LA, window prepared over the labial cortical bone in relation to the swelling revealing multiple denticles
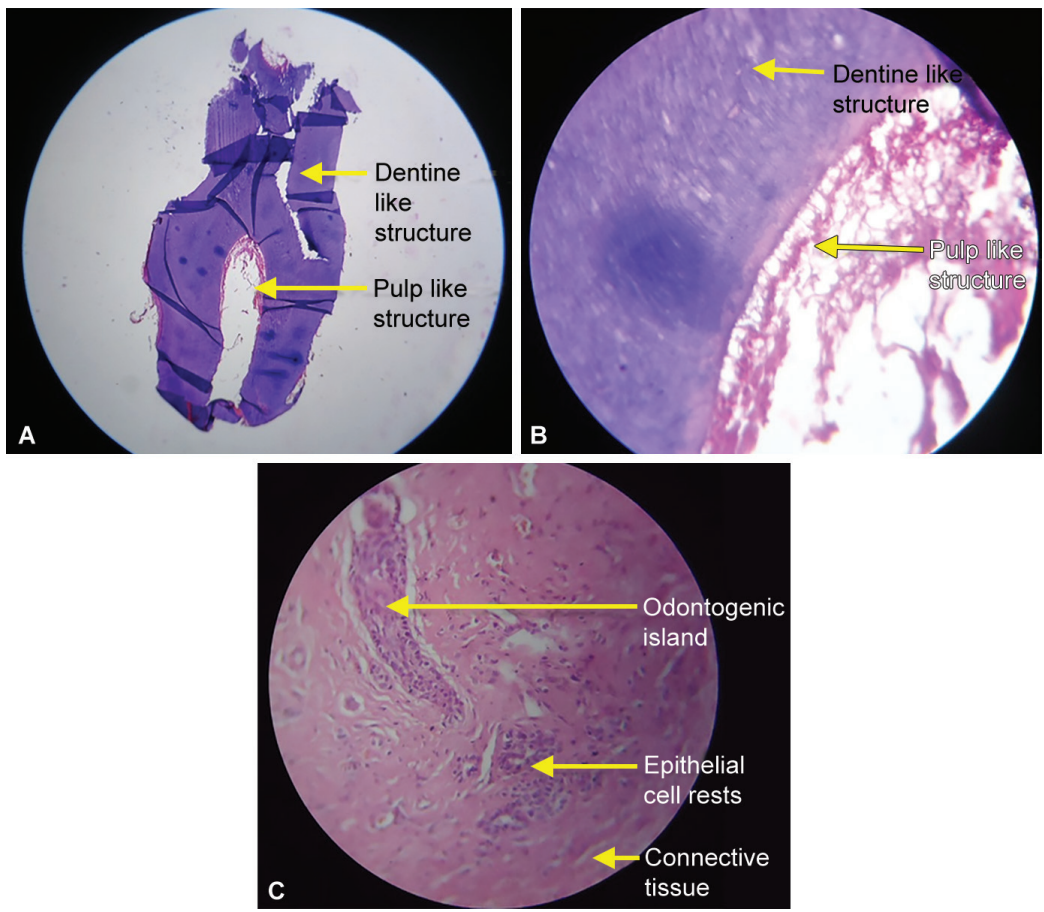

Figs 8 A to C: Histopathological examination: (A) Decalcified H\&E stain—under low power; (B) Decalcified H\&E stain—under high power; (C) H\&E stained tissue-under low power 

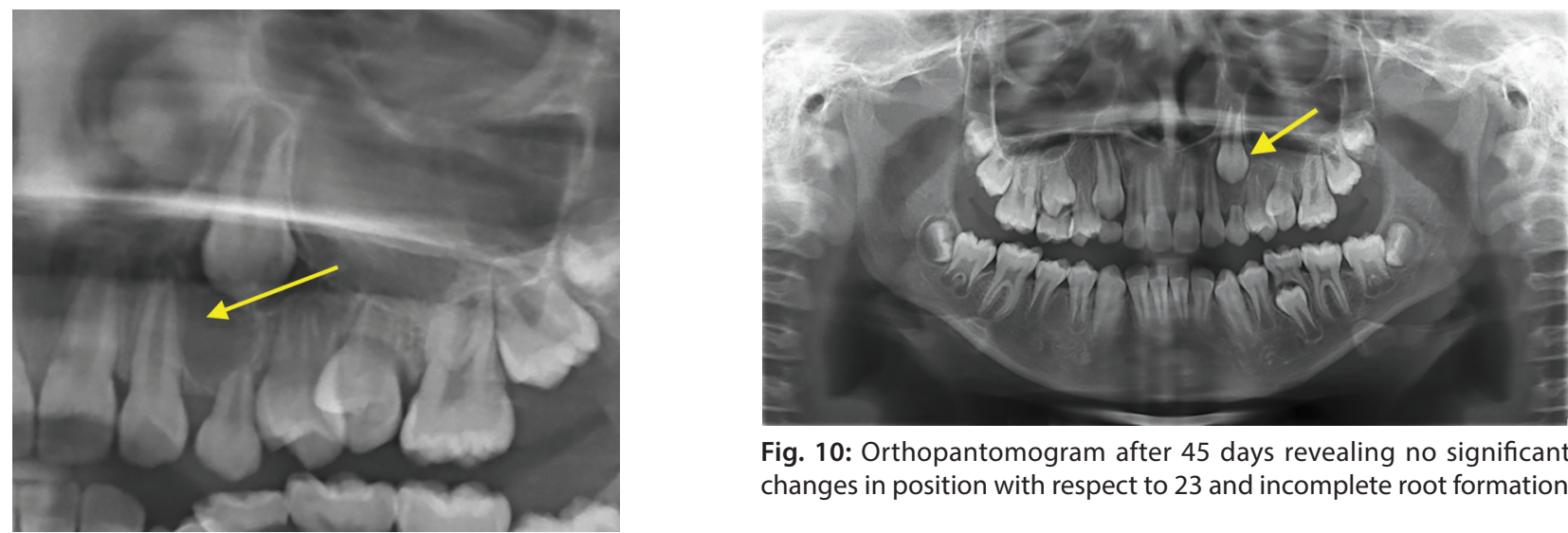

Fig. 10: Orthopantomogram after 45 days revealing no significant changes in position with respect to 23 and incomplete root formation

Fig. 9: Orthopantomogram after 7 days showing uneventful healing
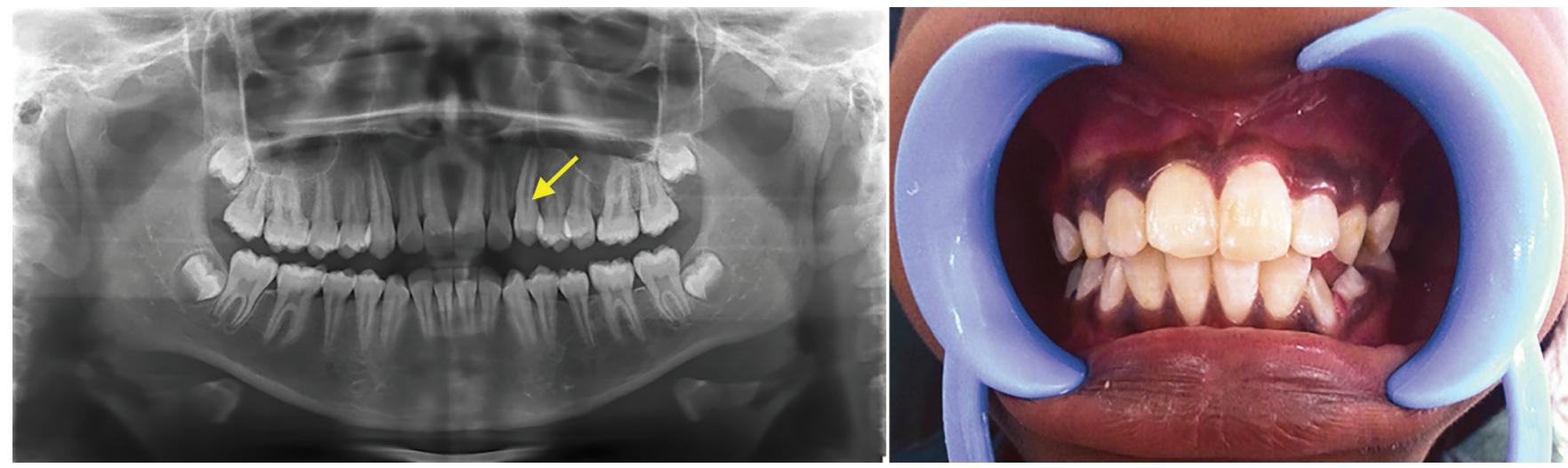

Fig. 11: Orthopantomogram after 11/2 years showing a normal trabecular pattern and healing at the surgical site and the canine has erupted into its normal position in the arch

Katz ${ }^{10}$ in review of 396 cases and Budnick ${ }^{9}(149$ cases) concluded that during the second decade of life odontomas were detected most frequently. The local hyperactivity of the dental lamina has been found to be the reason for the formation of the compound odontoma. ${ }^{11}$

Clinically, compound odontomas present with a painless, nonaggressive lesion, with a more limited growth potential than the complex odontoma but may cause impaction, retention of primary teeth, or delayed eruption of permanent teeth. They may be usually associated with pain, expansion of the cortical bone, and tooth displacement. Superficial odontomas cause expansion of the bone, which was observed in our patient. Swelling of the involved region and anesthesia of the lower lip can also be other symptoms. In this case, the patient reported with painless mild swelling of gums in relation to the left primary maxillary canine region and was diagnosed on the radiograph. The swelling was bony hard and nonfluctuant on palpation. Odontomas need to be differentiated from other painless swelling of jaws like osteoid osteoma, fibro-osseous lesions such as cemento-ossifying fibroma, and cementoblastoma. In this case only after clinical, radiological, and histopathological examination, we reached to the confirmatory diagnosis of the compound odontome. The accepted choice of treatment of the odontome is surgical exposure followed by enucleation of the odontome to allow for the eruption of the permanent tooth.

Reports by various authors concluded that the removal of the odontome leads to spontaneous eruption of the impacted tooth. ${ }^{12}$ Rapid eruption of the unerupted tooth can be achieved by the exposure of the impacted tooth during enucleation of the odontome followed by orthodontic traction using ligature/e-chain/ bonded attachments. ${ }^{13}$ The above invasive treatment approach can lead to discrepancy of the gingival level between the exposed tooth and its neighboring teeth resulting in inadequate gingival tissue attachment with poor gingival margin. ${ }^{14}$ In this case, the retained tooth 63 was left for natural exfoliation and the odontome alone was enucleated; the raised mucogingival flap was sutured back in position to allow for the eruption of the permanent canine physiologically.

Various studies suggest that recurrence of the odontome is rare once enucleated, but close monitoring in children is recommended. Vigilant follow-up of such cases both clinically and radiographically is required for assessing the eruption of the unerupted tooth. ${ }^{15}$ In some cases if the root formation of the unerupted tooth completes before eruption an interdisciplinary approach may be necessary, as the eruptive power is greatly diminished once root formation is completed. In our case, it was observed that the primary canine had exfoliated naturally and the permanent canine had physiologically erupted in position after 1 year and 6 months demonstrating that early diagnosis and treatment of an odontome ensures better prognosis. ${ }^{16}$

\section{Conclusion}

Clinical experience of the above case suggests that it is very important to correlate clinical and radiographic findings along 
with signs and symptoms for early diagnosis of the odontome that usually goes undetected. Early identification of odontomas facilitates uneventful eruption of the permanent teeth/physiologic eruption of the impacted permanent tooth, ensuring minimally expensive treatment and better prognosis.

\section{Clinical Significance}

Odontomas should be excised due to the presence of various tooth formulations that can predispose to cystic change, interfere with tooth eruption, and cause considerable bone expansion. Early removal of the odontomas allows the impacted tooth to restart the physiological eruption. The authors suggest that greater emphasis should be given on routine dental check-ups for children so that these anomalies can be detected earlier, thereby minimizing the interventions needed after enucleation.

\section{References}

1. Santosh BS, Anuradha V, Dayanand MC. Erupting complex odontoma: coronal to impacted second molar associated with dentigerous cyst. J Oral Health Comm Dent 2011;5(2):100-102. DOI: 10.5005/johcd5-2-100.

2. Dua N, Kapila R, Trivedi A, et al. An unusual case of erupted composite complex odontoma. J Dent Sci Rese 2011;2:1-5.

3. John JB, John RR, Punithavathy, et al. Compound odontoma associated with maxillary primary tooth: a case report. J Acquir Immune Defic Syndr 2010;1:49-51.

4. Kaban LB. Pediatric oral and Maxillofacial surgery. Philadelphia: Saunders; 1990. pp. 111-112.

5. Barbara LT, Campos DM, Nevarey Rascon MM, et al. Descriptive aspects of odontome: literature review. Rev Odont Max 2016;20(4):e265-e269.
6. Mosqueda-Taylor A, Ledesma-Montes C, Caballero-Sandoval S, et al. Odontogenic tumors in Mexico: a collaborative retrospective study of 349 cases. Oral surg Oral Med Oral Pathol Oral Radiol Endod 1997;84(6):672-675. DOI: 10.1016/S1079-2104(97)90371-1.

7. Neville BW, Damm DD, Allen CM, et al. Oral and Maxillofacial Pathology. Philadelphia: Saunders; 1995. pp. 531-533.

8. Ragalli CC, Ferreria JL, Blasco F. Large erupting complex odontoma. Int J Oral Maxillofac Surg 2000;29(5):373-374. DOI: 10.1016/S09015027(00)80056-9.

9. Budnick SD. Compound and complex odontomas. Oral Surg Oral Med Oral Path 1976;42:501-506. DOI: 10.1016/0030-4220(76)90297-8.

10. Katz RW. An analysis of compound and complex odontomas. ASDC J Dent Child 1989;56(6):445-449.

11. Philipsen HP, Reichart PA, Praetorious F. Mixed odontogenic tumours and odontomas. Considerations on interrelationship. Review of literature and presentation of 134 new cases of odontomas. Oral Oncol 1997;33(2):86-87. DOI: 10.1016/S0964-1955(96)00067-X.

12. Hidalgo-Sanchez O, Leco Berrocal MI, Martinez-Gonzalez JM. Meta analysis of the epidemiology and clinical manifestations of odontomas. Med Oral Patol Oral Cir Bucal 2008;13(11):E730-E734.

13. Batra P, Duggal R, Kharbanda OP, et al. Orthodontic treatment of impacted anterior teeth due to odontomas: a report of two cases. J Clin Pediatr Dent 2004;28(4):289-294. DOI: 10.17796/jcpd.28.4.f21 14718 u87u4712.

14. Hattab FN, Yassin OM, Rawashdeh MA. Supernumerary teeth: report of three cases and review of literature. ASDC J Dent Child 1994;61(5-6):382-393.

15. Das UM, Vishwanath D, Azhar U. A Compound composite odontoma associated with unerupted permanent incisor: a case report. Int $J$ Pediatr Dent 2009;2(2):50-55. DOI: 10.5005/jp-journals-10005-1030.

16. Zoremchhing $\mathrm{P}$, Joseph $\mathrm{T}$, Varma B, et al. A compound odontoma associated with unerupted permanent incisor-a case report. J Indian Soc Ped Prev Dent 2004;22:114-117. 